\title{
Mobile Tracking and Resource Reservation Scheme for Cellular Networks
}

\author{
Subbiah Shenbagaraman, B. Prabhakaran, S. Venkatesan \\ Department of Computer Science, \\ University of Texas at Dallas, \\ Richardson, USA, TX 75083 \\ Email: \{sxs014340, praba, venky\}@utdallas.edu
}

\begin{abstract}
Providing and maintaining Quality of Service (QoS) in cellular networks has been investigated by many researchers. Many schemes have been proposed and compared against each other. The main aim of all the approaches is to maximize the utilization of available resources like bandwidth. Location technology has helped widely in the conservation of resources. One of the algorithms that make use of the position of the mobile to reserve resources is the Shadow Clustering Scheme [2]. By tracking the mobile more accurately we can help any reservation protocol to reserve resources using its reservation algorithm. In this paper we propose a method to increase the utilization of resources by making more accurate predictions of the location of the mobile. This involves making use of a new mobile tracking scheme to determine the position of the mobile more accurately. This takes tracking the mobile to the intra cellular level as against the exiting tracking at cellular level. The information obtained form this mobile positioning scheme is then passed on to a reservation algorithm. Simulation of the proposed scheme upon two mobility models has been conducted and compared against reservation schemes that uses inter cellular tracking. The mean error in determining the position of the mobile is greatly reduced and also the accuracy with which we are able to predict the motion of the user is also increased. This increased accuracy helps in more accurate predictions and avoid unnecessary reservation of resources.
\end{abstract}

Keywords-component; mobile tracking; resource reservation; shadow clustering; location area;

\section{INTRODUCTION}

The deployment of wireless networks has been on the steady increase all over the globe during the last decade and is still expected to grow in the future however; the bandwidth spectrum that is available is finite. Knowing the position of a mobile station has a number of advantages to the service provider as well has numerous applications to the user. Mobile positioning has been used in the military for long and has been recently introduced into civilian radio applications. This led to introduction of location technology. This paper presents a new technique for resource reservation scheme for cellular networks using mobile tracking and also discusses the possible applications in mobile telecommunication network. Resources can be reserved in advance if we are able to predict the motion of the user using his current location. This requires mobile positioning. In our method the base stations transmit certain signals at different power levels that traverse different distances [1]. We use these to partition each cell into regions called Location Areas (LA), which lie in the intersection of various regions formed by these signals. The natural attenuation of the signals with distance is exploited to form the LA's.

\section{RESOURCE RESERVATION SCHEME}

A good QoS is expected of from any wireless network. The network should be able to support new calls as well as handle existing calls with their varying resource requirements as they move from cell to cell. As the mobile user moves from one cell to another, the call should be seamlessly handled with minimum delays for handoff, minimum packet loss and call drop probabilities. When a connection is accepted, the network should be able to support a minimum level of service to the user. This implies that a new connection can also be rejected to ensure the minimum level of service for already existing calls. The amount of such bandwidth that is reserved in the cells for calls that have already been accepted in the network always poses an optimization problem. We would be able to better optimize the reservation of resources if we are able to predict more accurately the path of the mobile.

Wireless networks provide communications services to a large number of mobile users. However, the spectrum of the available bandwidth is finite and should be multiplexed amongst the users. This requires efficient channel allocation methods to be used. The wireless networks should ensure continuous communication as the user moves form one cells to another while the communication is active. Location management comes into play when a user is to be tracked down to establish communication with that user. Mobile positioning can be one of the services offered by cellular network operators. Mobile location has played a role in wireless communication since its inception because cellular radio has an inherent positioning capability by the virtue of the fact that the mobile typically connects to the closest serving site. The typical method that is used for mobile positioning is based using triangulation techniques which use any of the parameter such as time, angle of arrival, time of arrival, power level of the forward or reverse link. In general two different types of location technique have been studied in order to locate a mobile. These are the network based solution and the handset based solution to position a mobile, nowadays a hybrid solution is being touted. 


\section{A. Mobile Tracking}

Tracing the path taken by a mobile user is mobile tracking. The first step towards mobile tracking is however mobile positioning. Mobile positioning requires the application to give the accurate latlong (latitude and longitude) of the mobile user with a margin of error, in mobile tracking; the error margin can be considerably large. By tracking the mobile we can know the path previously taken by the mobile and use it to predict the future trajectory on which the mobile user might move. This has many applications not only in emergency services such as E911, but also in tracking of fleet and material. On the system side, mobile tracking can aid in intelligent handoffs and QoS reservations for a mobile user in a network. On the application side, a host of new commercial applications called Location Based Services have also come up. These make use of the current location of the user and provide services that are specific to that location. Patients, pets and prisoners can also be tracked. Several techniques to locate the mobile station by measuring signal strength attenuation, direction of arrival, and delay of the radio signals exchanged between mobile station and multiple base stations. Positioning of a mobile is done by methods that involve time-of arrival (TOA) and angle-of-arrival (AOA) measurements. TOA methods require a minimum of three base stations while for AOA methods require only two base stations. The basic method of location estimation is to calculate the position of the mobile station trigonometrically. The distance of the mobile station can be used to calculate the position of the mobile station from three base stations from the received signal.

\section{B. Shadow Clustering}

The shadow clustering concept is used to estimate future resource requirements and to perform call admission decisions in wireless networks [2]. This is done by tracking the mobile as it moves along the cellular network. The QoS requirements of the mobiles users can be reserved in advance in neighboring cells for a call that has already been initiated. This method predicts the probability with which a mobile terminal $x$ currently in cell $j$ will move to cell $k$ at time $t$.

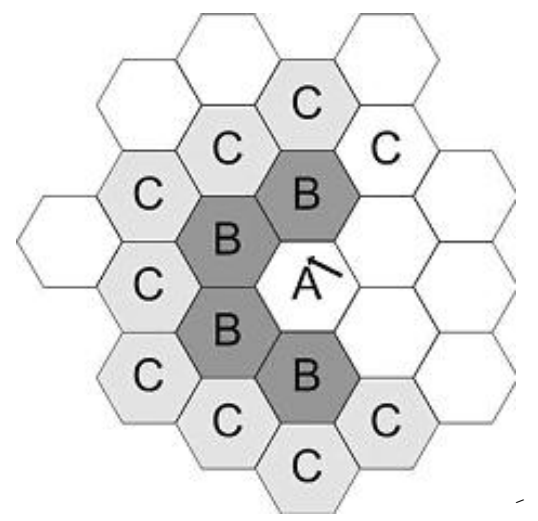

Figure 1. Shadow Cluster formed for a mobile in cell A

In Figure 1, the probability with which the mobile currently in cell A will enter any of the cell B is higher and shown darker than that of cells marked $\mathrm{C}$ whose probability is less. As the cell moves along the network, its shadow cluster keeps changing. The direction with which the mobile moves within a cell is not known and it direction from the past handoff and other statistical data is used to predict the direction or the future cells. The probability of taking a particular route is calculated with the initial handoff probability of mobile $x$ currently in cell $j$ would leave the cell through side $w$ and route $r$. The above mentioned scheme relies on tracking the mobile between the cells as it moves along the network. The mobile is not tracked as it moves within the cell

\section{Proposed RESOURCE RESERVATION SCHEME USING MOBILE TRACKING}

In our proposed scheme we rely on a method to track the mobile more efficiently within the cell so as to better optimize the reservation scheme which reserves resources based on the predicted motion of the cells. The proposed scheme increases the accuracy of the predicted motion by tracking the mobile not only as it moves between cells along the network but also within the cells as well.

\section{A. Proposed Model- 7 Cell Structure}

We use the 7 cell hexagonal cell model to explain the proposed scheme. In this model, all the cells are hexagonal in shape and six other hexagonal cells of equal size surround each interior cell. In figure 2 we see 7 cells numbered from 1 through 7. Each cell like cell number 1 would be surrounded by 6 similar hexagonal cells. Each cell has it own base station that is located at the center of each cell.

\section{B. Distance To Live Signals}

A base station is located at the center of each cell. The base station sends signals that carry distance information, and these signals are transmitted with different power levels [1]. Note that transmission power determines transmission range. We call these signals distance-to-live signals (DTL). These are conceptually similar to time-to-live (TTL) packets in computer networks. Each signal that is sent by the base station has information that identifies the base station from which the signal originated and also information about the distance of the signal from the center of the cell, this can also be any other identifier that is able to identify this signal uniquely during processing to obtain the location information. In the proposed model we assume the signal to have information such as a unique id for the base station from which it receives the DTL signal, distance information and other identifiers. A base station sends several such signals and the number is determined based on the factors such as granularity, load on the network, topology, and required accuracy for mobile positioning. The signals need not be continuous signals but can be multiplexed over the same channel by varying the signals over time. In this process we do not waste bandwidth for mobile positioning that could otherwise be used for carrying data over the network. The time interval between these multiplexed signals should be chosen such that the signals appear to be continuous to the user. 


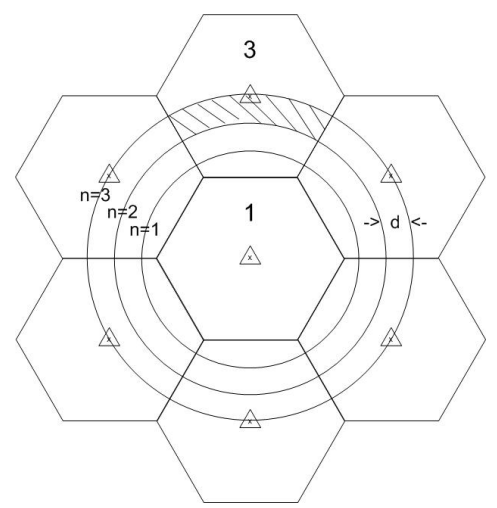

Figure 2. DTL signals

Figure 2 shows three such DTL signals of base station 1, that are in the neighboring cells surrounding cell 1 . Consider the base station of an inner cell. The reachability of the different signals can be represented by concentric circles centered at its base station. These circles define the bands that determine the area between the circles. The power level of the DTL signals is adjusted in a manner that the outermost signal, which forms the last circle, is big enough to reach each of the six adjacent base stations. Thus each base station has its own set of DTL signals. The DTL signals carry information such as the id of the base station that transmits it and the distance information. The distance to which a signal lives can be controlled by varying its power based on various factors, which should also include topology of the cell and multipath fading. The shaded part represents the area of cell 3 where the 3rd DTL signal of cell 1 (with highest power of cell 1) can be received but the other 2 DTL signals of cell 1 cannot be received. By varying $n$, the width of the shaded region can be varied. In order to subdivide the shaded area for finer location determination, DTL signals transmitted by cells 2 and 4 can be used.

\section{Location Area Concept}

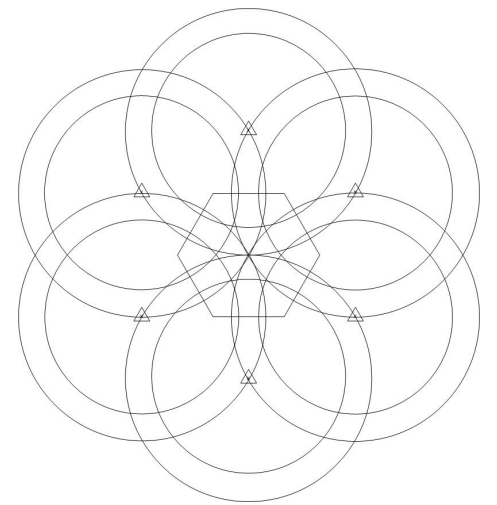

Figure 3. LA's formed in a cell by DTL signals of neighboring cells

Each cell is divided into location areas by the overlapping DTL signals and the whole coverage area of a cell mapped into location areas. If each cell transmits 2 DTL signals, Figure 3 shows their intersection at cell 1 . (The intersection of signals at other cells is not shown for clarity.). The DTL signals of each base station overlap with the DTL signals of the neighboring cells and the intersection of these signals form a location area. Each LA thus formed is bounded by the signals and hence can be uniquely identified. The LA can be bounded by signals and also by the boundary of the cell. Thus each LA has its unique set of identifiers which may be DTL signals or a combination of DTL signals and the cell boundary. This is used to identify the location of the cell.

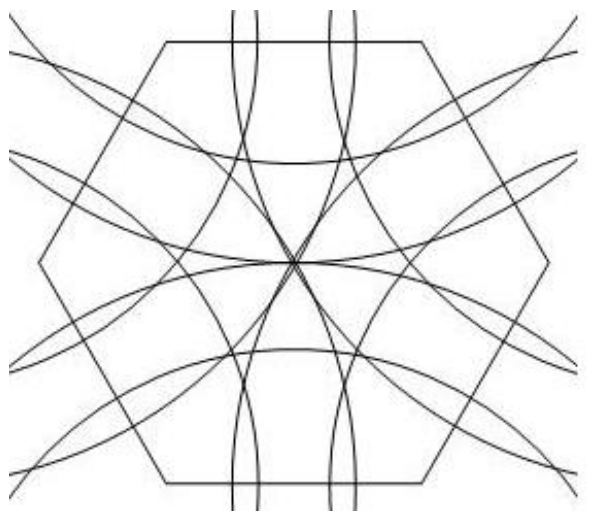

Figure 4. Location Area formed in a cell by DTL signals

The matrix is an $l * m$ matrix, where $l$ and $m$ are two more than the maximum of the number of location areas along the length or breadth of the cell respectively. Note that we add two more since LAs from the neighboring cells can be used for continuity. The path taken by the mobile can be recorded (on the matrix) and traced whenever needed [1]. The distance of the neighboring base stations from each location area is maintained at the serving base station. When a mobile is located at a particular location area, the probability with which it can enter the next cell can be determined. The probability with which a movement of a mobile can be predicted has been suggested in various proposals. User profiles are made used [7]. Random walk is more useful for pedestrian movement in which mobility is generally confined to a limited area. The authors of [8] have used this model for location management in wireless networks. Fluid flow model is suitable for users with high mobility and has been discussed in [9].

\section{Location Areas as a Matrix}

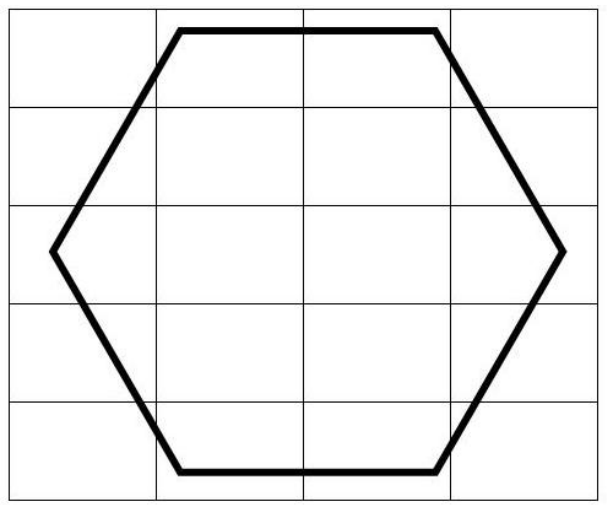

Figure 5. Location Areas as a matrix 
There are $\mathrm{k}$ location areas that surround each location area. The direction of the mobile will help reduce the probability of the movement of the mobile in a region of $[0, \pi]$ around the $\mathrm{k}$ location areas. The probability with which a mobile will move will largely be predicted based on its direction, the direction in which the mobile travels can be determined from the last $y$ location areas visited. For any value of $y$ only finite number of orientations of the $y$ location areas is possible. These finite orientations determine the direction in which the mobile is moving. This number of orientations is further reduced if the topology of the location area is known. Note that we can also determine the rate of change of location (speed of the mobile). By tracking the mobile more accurately within the cell we would be able to make a more accurate prediction in the path of the mobile. Using these predictions in a resource reservation scheme would optimize the reservation of resources.

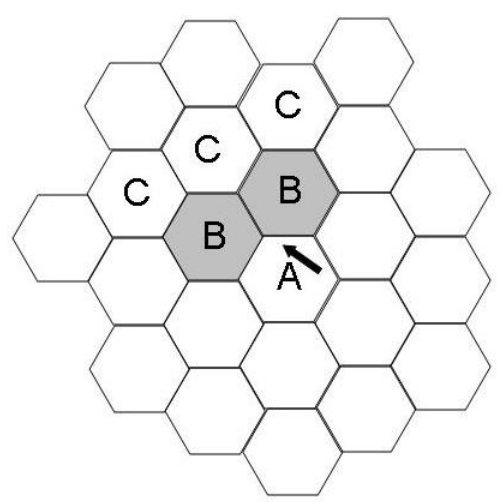

Figure 6. Enhanced Shadow Clustering

The resource reservation scheme used is the Enhanced Resource Reservation Scheme [1]. Figure 6 shows an enhanced shadow cluster that is formed for a cell in the neighboring cells. The number of resources reserved can be reduced by more accurate prediction of the mobiles. The shadow cluster of the mobile keeps changing as the mobile moves along the network. The direction of the mobile as it moves within the cell is also known as we are tracking the mobile within the cell with the LA's. Using the direction, velocity and statistical information the next position of the mobile after a unit of time interval can be predicted. Prediction accuracy is greatly improved by tracking the mobile within the cell and knowing the direction of motion of the mobile. This increase the accuracy reduces the number of cells that are included in the shadow cluster of the mobile and hence more valuable resources that might otherwise be blocked is freed up. The proposed method enhances the efficiency of the shadow clustering method by reducing the number of cells in the shadow cluster as the mobile can be traced within a cell.

In any mobile radio system, the transmitted RF signal is subject to a number of propagation effects before reception. These include a strong path loss component and two significant fading components. Path loss is due to the physical separation of the radios whilst the fading is caused by the diffraction and reflection of the signal energy due to terrain and any natural or man made obstructions. The two fading components are generally referred to as fast and slow fading. The impact of these propagation effects on mobile systems has been the subject of extensive study and research over the past years. For mobile positioning, the most significant effect due to signal propagation is multipath, which each path arriving at different times and angles. The different arrival times are normally characterized by a parameter known as delay spread. In the proposed system, the degradation of the signals due to fading may cause a mobile to report signals that in error. This would make a mobile to appear in a different LA than the one that it is currently in. This can be overcome by using the signals of the serving base station to position the mobile. This allows the mobile to be positioned correctly even if one of the signals received by the mobile is in error.

\section{Simulation Results}

For performance evaluation we have considered a cellular network where there are 50 cells in the coverage area, the radius of the cells is assumed to be $500 \mathrm{~m}$. The simulation was done using two different mobility models and we calculate the probability distribution of the physical location of the user at a given time $t$, provided that we know his or her location at a previous time $t_{0}<t$. All users in our simulation move towards a destination chosen from a uniform distribution, the speed of the mobile also varies for each destination within the coverage area. The short distance variable or segment length, $l$ is varied to study the behavior of the system.

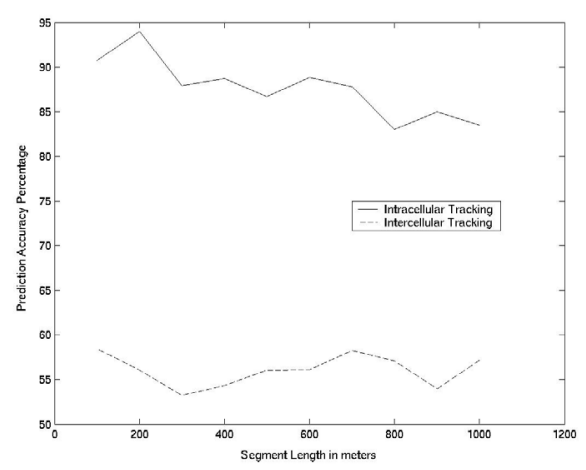

Figure 7. Prediction Accuracy Percentage versus Segment Length for mobilty model 1

A node is described by its current space vector $(x(t), y(t))$, its current speed $v(t)$, and its current destination the coordinate that lies within the space defined by $(0,0)$ and $(\max (X)$, $\max (Y))$, the path taken by the point. The velocity with it travels $\mathrm{v}$, is also. In our first simulation model, we evaluate the prediction accuracy percentage for various segment lengths for the mobility model described. As the mobile moves along the coverage area within the coordinate $(0,0)$ and $(\max (X)$, $\max (Y))$, the path taken by the mobile is recorded at the LA level, the exact coordinates are not noted. The prediction accuracy is compared when the mobile is tracked at intercellular level against intracellular level. 
- The maximum accuracy with we can predict the position of a mobile in a LA for our assumptions varies from $82 \mathrm{~m}$ to $110 \mathrm{~m}$.

- The predictions of the mobile user location given by our algorithm have mean error in the range of $38 \mathrm{~m}$ to $90 \mathrm{~m}$.

- In the proposed scheme, when the segment size is small, the prediction accuracy is higher. For example when the segment size is between $0 \mathrm{~m}$ and $200 \mathrm{~m}$, the prediction accuracy is about $95 \%$. As the segment size increases the prediction accuracy decreases and later stabilizes to about $80 \%$.

- Without the proposed scheme, for small segment sizes the prediction accuracy is $40 \%$ and as the segment size increases the prediction accuracy also increases and stabilizes to about $60 \%$.

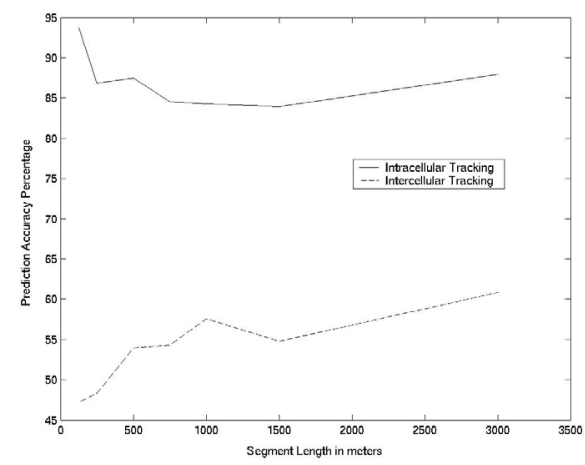

Figure 8. Prediction Accuracy Percentage versus Segment Length for mobilty model 2

\section{CONCLUSION}

When the location of the mobile is known, it can be used within the wireless system to improve the system performance in the different layers for system enhanced applications and increase wireless system functionality for location based commercial services. System Level Applications - Location information can be used to improve system performance. Some example applications which could make us of location information are listed below.

1. Intelligent Handoff System uses signal strength and MS location information to reduce unnecessary dropped calls as well as maintain quality of service [11].

2. Efficient Channel Allocation Using location information we could determine the velocity and dynamically allocate channel capacity by reserving channels in the target cell for each user [14].

3. Commercial Location Based Services. Tracking ServicesFleet management allows timely allocation of resources which helps reduce response time to new calls. Car navigation linking with the map portal and also provide real time users current position and surrounding information.

4. Value Added Services - Mobile station requests the nearest ATM, cheapest gas station, local traffic information etc.

5. Messenger Services - Network notifies the user's current location to friends and also provides the location of friends to the user

\section{REFERENCES}

[1] Subbiah Shenbagaraman, S. Venkatesan, B. Prabhakaran, "Mobile Tracking using Forward Link in Cellular Networks." Proceeding of IEEE Emerging Telecommunications and Technologies Conference, September 2002.

[2] David Levine, Ian F.Akyildiz, Naghshineh, A Resource Estimation and Call Admission Algorithm for Wireless Multimedia Networks Using the Cluster Concept. IEEE/ACM Trans. On Networking, Vol 5, No.1, Feb 1997

[3] L.Hsu, R.Purnadi and S.Wang, "Maintaining Quality of Service(QoS)during handoff in Cellular System with Movement Prediction Scheme ,'IEEE VTC-99 Fall, Sep15-19, 1999.

[4] H. L. Song, Automatic vehicle location in cellular communication systems, IEEE Trans. Veh. Technol., vol 43 , pp.65-71,Feb. 1997.

[5] M. Hata and T.Nagatsu, Mobile location and signal strength measurement in a cellular system, IEEE Trans. Veh. Technol., vol VT29 May. 1980.

[6] F. Cesbron and R. Arnott, Locating GSM mobiles using antenna arrays, Electron. Lett ., vol. 34, no. 16, pp. 1539-1540, Aug. 1998.

[7] J. S. M. Ho and I.F. Akyildiz, "Mobile user location and paging under delay constraints," ACM - Baltzer J.Wireless Networks, vol 1., Dec. 1995.

[8] Y-B Lin, " Reducing location update cost in a PCS network," IEEE/ACM Trans. Networking, vol. 5, pp. 25-33, Feb. 1997.

[9] J.G Markoulidakis, G. L.Lyberopolous and M. E. Anagnostou, "Traffic Model for third generation cellular mobile telecommunication systems," ACM - Baltzer J.Wireless Networks, vol 4., Aug.1998.

[10] J. Caffery Jr. and G. L. Stuber, Subscriber location in CDMA cellular networks, IEEE Trans. Veh. Technol., vol 47 ,pp. 406-416, Nov. 1998.

[11] M.Green and S.S. Wang, "Soft Decision Handover based on Signal Strength and Mobile Location", IEEE VTC-2000 Fall, APr. 9-14, 2000.

[12] Bill N.Schilit, Marvin , Brent B.Welch, "Customizing Mobile Applications," USENIX Symposium on Mobile and Location Independent Computing.

[13] Silventonien M. I and Ratalainen T, Mobile station emergency locating in GSM, IEEE International Conference on Personal Wireless Communication, pp. 232-238, 1996 . 